\title{
Geometry of the Cosmic Web: Minkowski Functionals from the Delaunay Tessellation
}

\author{
Miguel A. Aragón-Calvo \\ Department of Physics and Astronomy \\ The Johns Hopkins University, \\ 3701 San Martin Drive \\ Baltimore, MD 21218, USA \\ miguel@pha.jhu.edu
}

\author{
Sergei F. Shandarin \\ Department of Astronomy \\ The University of Kansas \\ Lawrence, KS 66045, USA \\ sergei@ku.edu
}

\author{
Alexander Szalay \\ Department of Physics and Astronomy \\ The Johns Hopkins University, \\ 3701 San Martin Drive \\ Baltimore, MD 21218, USA \\ szalay@jhu.edu
}

\begin{abstract}
We present a novel method for computing the Minkowski Functionals from isodensity surfaces extracted directly from the Delaunay tessellation of a point distribution. This is an important step forward compared to the previous cosmological studies when the isodensity surface was built in the field on a uniform cubic grid and therefore having a uniform spatial resolution. The density field representing a particular interest in cosmology is the density of galaxies which is obtained from the highly nonuniform distribution of the galaxy positions. Therefore, the constraints caused by the spatially uniform grid put severe limitations on the studies of the geometry and shapes of the large-scale objects: superclusters and voids of galaxies. Our technique potentially is able to eliminate most of these limitations. The method is tested with some simple geometric models and an application to the density field from an N-body simulation is shown.
\end{abstract}

Keywords-Delaunay Tessellations; Minkowski Functionals; cosmology

\section{INTRODUCTION}

Redshift surveys of galaxies have shown that even on the largest observed scales the bulk of matter in the universe is concentrated in clusters and superclusters of galaxies which are separated by huge almost empty regions, called voids of galaxies. Cosmological N-body simulations in the currently popular $\Lambda \mathrm{CDM}$ model reveal that at moderate density thresholds $\delta \sim 3$, the supercluster network percolates, while occupying a small fraction $(\sim 3 \%)$ of the total volume [32]. At greater density thresholds the structure breaks into disconnected parts most of which are clusters of galaxies and long filaments that link several clusters of galaxies together similar to structures shown in Fig. 4. They are often referred to as superclusters of galaxies. At even greater thresholds only the clusters of galaxies are seen as islands in a sea.

A number of statistical measures have been suggested to quantify the patterns made by spatial concentrations of galaxies. The traditional approach to quantify clustering makes use of the hierarchy of correlation functions [24]. Unfortunately they become cumbersome to evaluate beyond the three point function and are not particularly revealing as geometry and shapes are concerned. Various statistics were proposed to quantify the geometrical and topological properties of the structure: e.g. percolation analysis [30], [48], counts in cells [15], [17], minimal spanning tree [4], the genus measure [12], wavelet analysis [18], Multiscale Morphology Filters from the Hessian of the density field [1] and potential field [14], and recently the SpineWeb algorithm [2] and the skeleton of the cosmic web [21], [39], etc.

Mecke, Buchert and Wagner (1994) have introduced the Minkowski functionals (hereafter MF) to cosmology. The four Minkowski functionals computed for the excursion sets at many density thresholds contain valuable information regarding both the geometrical as well as topological distribution of matter in the Universe. There have been three major attempts made to study the morphology of the Cosmic Web using MF; these efforts differ in their approach of evaluating the MF: (1) Firstly, Boolean grain models study the MF of surfaces which result due to intersecting spheres decorating the input point-set [20]. (2) Secondly, Krofton's formulae make it possible to calculate MF on a density field defined on a grid [29]. In this case the MF are calculated by using the information of the number of vertices, edges, faces and cuboids. (3) Finally, an alternative, resolution-dependent approach consists in employing the Koenderink invariants [29].

A radically new and powerful approach for using the Minkowski functionals in cosmology was firstly introduced for the analysis of two-dimensional CMB (Cosmic Microwave Background) maps [22], [33]. This approach consists of constructing isotemperature contours and analysis of individual parts of the excursion set at many levels. These contours are triangulated and the MF are evaluated for the resulting closed polygons. Since Minkowski functionals are additive in nature one can glean information regarding both individual objects (galaxies, clusters, voids) as well as the supercluster-void network in its totality.

However, a three-dimensional algorithm required a new component that builds a surface of approximately constant (to linear order) density. It was implemented in [37] and then used for the analysis of the mass distribution at the 
non-linear stage in cosmological N-body simulation of the $\Lambda$ CDM model [34] and mock galaxy catalogs [35], [36]. In addition, particular ratios of Minkowski functionals quantifies the morphology of large scale structure by telling us whether the distribution of matter in superclusters/voids is spherical, planar, filamentary etc. [25]. The numerical technique used in the cosmological studies is based on the marching cubes algorithm (MCA) in three-dimensional density field specified on a uniform mesh. It therefore has a uniform resolution scale over the region in question. The data points are the positions of the galaxies which are distributed in highly non-uniform manner: there are clusters of galaxies where thousands of galaxies packed in the volume of a few $\mathrm{Mpc}^{3}$ and there are voids of galaxies where hardly one galaxy can be found in hundreds of $\mathrm{Mpc}^{3}$. Thus, for the study of the densest clusters of galaxies one needs to have a fine mesh, but it does not work in voids were most of sites are empty. On the other hand a mesh with large cells would allow to study the voids but erase the clusters. A numerical method based on adaptive mesh is badly needed for the studies of the structure in the universe.

\section{A. Extracting implicit surfaces from scalar fields}

The extraction of implicit surfaces from a given scalar field is an important problem for visualization and volumetric data analysis. The marching cubes algorithm [16] was introduced in order to extract isosurfaces from a regular grid by identifying intersections between each voxel of the grid and an isosurface defined by a threshold value $\delta_{t}$ and then generating a triangular mesh from the intersecting points. The MCA is an efficient and fast solution but it presents a few ambiguities. In addition to this its use was until recently restricted by a patent. In order to circunvent the patent restrictions and solve the ambiguities in the MCA the Marching Tetrahedra Algorithm (MTA) was developed [10], [13]. The main idea behind MTA is to divide each voxel of the regular grid into 6 tetrahedra and identify the intersections between each tetrahedron and the isosurface defined by $\delta_{t}$. This greatly simplifies the identification of intersections since there are only 8 cases to consider: no intersection, 4 intersections with a triangle and 3 intersections with two planar triangles or quad (see Fig. 1). There are no ambiguities in the intersection cases and they can be conveniently encoded in a lookup table. Both MCA and MTA use linear interpolation in order to identify the intersecting points.

The MCA and MTA take as input a scalar field sampled on a regular grid. In many of the applications of MCA and MTA the scalar field is computed directly in a previous step, like in tomography and MRI medical images or analytic functions for 3D texture and surface generation in computer images. In the astronomical context often the scalar field (density) must be reconstructed from a point distribution such as mass particles from N-body simulations or galaxy positions from redshift surveys. The accurate reconstruction of such datasets is not trivial an in general one has to rely on a series of assumptions about the underlying scalar field such as continuity and linearity.

A major improvement in the reconstruction and analysis of density fields from point distributions came with the implementation of Voronoi and Delaunay tessellation techniques [7], [9], [11], [23], [45]. In particular for astronomy the Delaunay Tessellation Field Estimator (DTFE) [27], [28], [44] based on the previous work of [5] which applied the Delaunay tessellation for the interpolation of a field sampled by an (in principle) arbitrary point distribution. The DTFE estimates local densities at each point as being inversely proportional to the volume of the adjacent Voronoi cell of the point. The density field is then sampled on a regular grid by linearly interpolating the density field inside each tetrahedron containing the sampling point. The DTFE represents a natural alternative to grid-based methods as it adapts itself to the point distribution, it produces a spacefilling density field and is well defined for all regions inside the volume containing the points. However, the DTFE is also known to produce strong artifacts as a result of the linear interpolation of the density field over very large and thin triangles. By construction it can not provide zero density estimates, therefore it can not reproduce the density field inside very underdense regions where there are no sampling points. In its original implementation it samples the density field on a regular grid thus the final density field is not adaptive and due to the discrete sampling scheme it introduces aliasing in the density field.

The use of a regular grid is not required in the case when we already have values of the scalar field at each sampling point. By recognizing that the Delaunay interpolation schema does not require the coupling with the point distribution (as in the DTFE) one can use the tessellation to interpolate any scalar field independently of the sampling points. Here we use a Delaunay tessellation to describe the connectivity between points that will be used to interpolate the density and extract isosurfaces. The density field can be computed by adaptive methods such as the scale-adaptive DTFE, Spline or fixed-scale Gaussian kernel. The density values at each vertex of the tessellation are then used to identify intersections with the isosurface. By computing the isosurfaces directly from the Delaunay tessellation we avoid two extra steps: i).- interpolation of the density field on a regular grid and ii).- tetrahedrisation of the voxels in the grid. Our algorithm can be directly applied to the Delaunay tessellation with no further processing.

\section{ISOSURFACES FROM THE DELAUNAY TESSELLATION: MARCHING TETRAHEDRA Algorithm}

The first step in the reconstruction of isosurfaces is the computation of the Delaunay tessellation of the point 
distribution which will be used to define the spatial connectivity between points used to interpolate the density field. The tessellation is also used to generate adaptive density estimates at each point as described in [27]

\section{A. Isosurface extraction}

Once we computed the Delaunay tessellation we test each tetrahedron for intersection with the isosurface defined by the threshold value $\delta_{t}$. We do this by simply evaluating $\min \left(\delta_{i}\right) \leq \delta_{t} \leq \max \left(\delta_{i}\right)$, where $\delta_{i=0 \ldots 3}$ is the density field evaluated at each of the four vertices of the tetrahedron. We then proceed to count the number of edge intersections. There are 7 intersecting cases (see Fig. 1), when the isovalue divides the tetrahedron in two lower values and two higher values the tetrahedron is cut by a quad, otherwise it is cut by a single triangle. Each intersecting point corresponds to a vertex of the triangulated isosurface. The position of the intersecting point is estimated by interpolating the field between the two vertices $\left(\mathbf{x}_{\mathbf{0}}, \mathbf{x}_{1}\right)$ of the intersected edge as follows:

$$
\begin{aligned}
& x=(1-t) x_{0}+t x_{1} \\
& y=(1-t) y_{0}+t y_{1} \\
& z=(1-t) z_{0}+t z_{1}
\end{aligned}
$$

where the parameter $t$ runs in the interval $[0,1]$ and is given by:

$$
t=\left(\delta_{t}-\delta_{0}\right) /\left(\delta_{1}-\delta_{0}\right) .
$$

Depending on the number of intersecting points we construct a triangle ( 3 intersections) or pair of coplanar triangles for a quad (4 intersections). The vertices of the triangle are then oriented to have their normal vector pointing outside the surface i.e. in the direction of decreasing density. This can be easily checked with the inner product between the normal of the triangle and the less dense vertex of the tetrahedron. The orientation of the quads is also checked to avoid incorrect triangulations when the vertices are not properly oriented as adjacent triangles.

\section{IMPLEMENTATION}

The Delaunay tessellation of the point distribution was computed using the publicly available CGAL (www.cgal.org) library. This efficient library allows us to compute the tessellation for $256^{3}$ particles in a couple of minutes on a regular linux workstation. Our implementation can generate open or periodic boundary conditions by using a buffer region around the original box and replicating the indexes of each point. Note that the most recent CGAL release includes periodic boundaries natively. This new feature will be included in the next version of our code. For the present application our approach is sufficient.

The code that constructs the isosurface from the Delaunay Tesselllation was written as part of a larger visualization
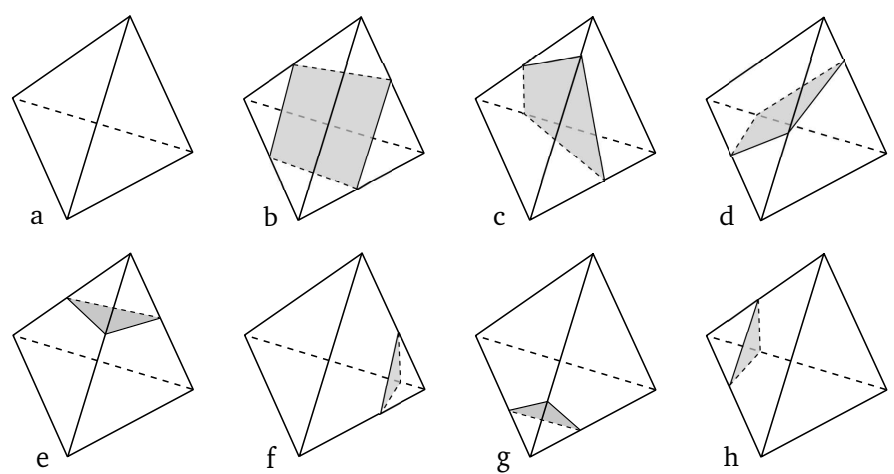

Figure 1. Possible intersection cases between a tetrahedron and the isosurface. a: no intersection with tetrahedron, b-d: intersection in a quad, there are two more dense vertices and two less dense. e-h: intersection in a triangle, there is one single denser or less dense vertex.

suite written in $\mathrm{C}++$ built on top of the OpenFrameworks (http://www.openframeworks.cc) environment for OpenGLbased volume and surface renderings as well as multimedia mixing for data sonification, sound visualization, etc. (see Fig. 2).

A version of the MTA was also written in the IDL language for fast development and testing of the algorithms. The code loops over all tetrahedra and constructs triangles or quads as described in section III. This raw surface consists of unconnected triangles so we proceed to remove duplicated vertices by indexing them. Repeated vertices are identified by means of the edge from which they were created. Al vertices sharing the same parent edge correspond to the same point.

\section{A. Isolated Object Extraction}

In general, the isosurface consists of a set of isolated objects. In order to study the properties of each individual object we must be able to identify it. This is done by first constructing a list of adjacent triangles. For each triangle in the isosurface we iteratively link and label its three adjacent triangles until all triangles are connected. The final triangulation divided into isolated objects is stored in a binary format and as a Waveform .obj format file that can be used for analysis and visualization.

\section{B. Density estimation}

In order to interpolate the density field inside each tetrahedron we need density estimates at each point. There are several methods for this purpose, among them the n-nearest neighbor, top-hat counts, Gaussian kernel and DTFE are the most common [5], [38], [42]. Adaptive density estimators such as the DTFE are strongly dependent on the spatial sampling. This is more evident in low density regions where one can see strong artifacts arising from the linear interpolation inside the tetrahedron. The use of averaging procedures on the tessellation or higher order interpolation alleviates 
some artifacts at the expense of increased complexity of the method. The end result is that isosurfaces extracted from adaptive density estimates tend to be highly irregular in low density regions. For the analysis presented in the coming sections we choose a fix-scale Gaussian kernel density estimator. This limits out ability to probe into compact high density regions such as the interior of clusters. However, for low-intermediate density regions the Gaussian smoothing produces better surfaces than the adaptive approaches like the DTFE provided that the smoothing kernel is larger than the mean interparticle separation. By using a Gaussian kernel we still take advantage of the space-filling properties of the tessellation and the convenient use of tetrahedra to interpolate the density field and identify intersections with the isosurface. In a following paper we will present a more detailed comparison between adaptive and fixed-scale density estimation. For the purposes of this work we restrict our analysis to Gaussian densities.

We compute Gaussian densities at particle $i$ as the sum of a Gaussian kernel evaluated at each of the surrounding $j$ particles:

$$
\rho_{i}=\sum_{j} e^{-\left(x_{j}-x_{i}\right)^{2} /\left(2 \sigma^{2}\right)}
$$

where $\sigma$ is scale of the smoothing kernel. For convenience we use the overdensity defined as:

$$
\delta=\frac{\rho_{i}}{\hat{\rho}}
$$

Where $\hat{\rho}$ is the mean density computed for the given $\sigma$.

The density estimation is an $O\left(N^{2}\right)$ operation, which makes the brute-force implementation unfeasible for large datasets. We use a bucket algorithm where the containing box is divided into a coarse grid. Instead of evaluating the Gaussian kernel for all the particles we restrict the operation to particles inside bucket containing particle $i$ as well as its 26 adjacent buckets. We use a grid size such that there is a warranty of enclosing particles up to $5 \sigma$ away from the target particle. Our fully threaded code greatly improves the speed of the density estimation and scales almost linearly with the number of processors.

The isodensity surfaces computed assuming a linear variation of the density field inside each tetrahedron tend to be very irregular. This effect is even more pronounced in the interface regions between high and low density cosmological structures such as the edges of walls, filaments and clusters where the density field profile is close to a power law. In these regions, if the spatial sampling is low, there can be a large variation in the density field inside an individual tetrahedron. Also, the overall density distribution of the evolved Cosmic Web is roughly log-normal [8]. As a result of this, the isodensity surfaces computed from linear interpolation tend to be highly irregular and to overestimate the enclosed volume inside the surface. Based on the character of the density distribution and the power-law behavior of the density profiles it makes more sense to use a transformation that linearizes the field inside the tetrahedron. We therefore use the $\log (\delta)$ and linearly interpolate the log-transformed density field. The $\log (\delta)$ interpolation does not conserve mass as in the linear case but it produces smoother surfaces and better volume estimation.

\section{Morphological Parameters}

\section{A. Minkowski Functionals}

In this work we discuss the geometry and topology of the regions bounded by the isodensity surfaces and therefore make no prior assumptions about the shapes of superclusters and voids. It is worth noting that some regions may have more than one boundary surface and possess nontrivial topology of the boundaries. The complete characterization of an arbitrarily complex region in three dimensions obviously cannot be achieved if only a few numbers are used. At best one can try to design some basic characteristics that serve a particular purpose. Our purpose is to provide basic measures suitable for quantification of typical components of the largescale structure: superclusters and voids.

Four Minkowski functionals are effective non-parametric descriptors of the morphological properties of surfaces in three dimensions [19], [20], [37]. They are

- Volume $V$ enclosed by the surface $S$,

- Area $A$ of the surface,

- Integrated mean curvature $C$ of the surface,

$$
C=\frac{1}{2} \oint_{S}\left(\frac{1}{R_{1}}+\frac{1}{R_{2}}\right) d a,
$$

where $R_{1}$ and $R_{2}$ are the principal radii of curvature at a given point on the surface.

- the Euler characteristic

$$
\chi=\frac{1}{2 \pi} \oint_{S}\left(\frac{1}{R_{1} R_{2}}\right) d a .
$$

\section{B. Shapefinders}

As demonstrated in [25], [26] particular ratios of Minkowski functionals called shapefinders provide us with a set of non-parametric measures of sizes and shapes of objects. Therefore, in addition to determining MFs we shall also derive the shapefinders, $T$ (Thickness), $B$ (Breadth) and $L$ (Length) defined as follows:

$$
T=\frac{3 V}{A}, \quad B=\frac{A}{C}, \quad L=\frac{C}{4 \pi} .
$$

This normalization gives roughly a half of the diameter in the case of anisotropic objects. The three shapefinders describing an individual region bounded by one or several isolated surfaces of constant density have dimensions of length and provide us with an estimate albeit quite crude of the region's 'extensions': $T$ is the shortest and thus describes the characteristic thickness of the region or object, $L$ is 
typically the longest and characterizes the length of the object; $B$ is intermediate and can be associated with the breadth of the object. This simple interpretation is obviously relevant only for fairly simple shapes. The choice of the coefficients in (7) results in a sphere having all three sizes equal to its radius $T=B=L=R$. A triaxial ellipsoid has values of $T, B$ and $L$ close but not equal to the lengths of its three principal semi-axes: shortest, intermediate and the longest respectively. It is worth noting that $T, B$ and $L$ are only the estimates of three basic sizes (semi-axes) of an object which work quite well on such objects as a triaxial ellipsoid and torus [25], [26], [37] but no three numbers can describe an arbitrary, complex three-dimensional shape. In particular, the length of a region with many tunnels may be better characterized by $\tilde{L}=L /(1-\chi / 2))$, because $(1-\chi / 2)$ is equal to the number of tunnels and therefore $\tilde{L}$ approximately represents the length of the parts between tunnels.

As suggested in [25] a rough idea about the global 'shape' of a region can be provided by an additional pair of dimensionless shapefinders:

$$
P=\frac{B-T}{B+T} ; \quad F=\frac{L-B}{L+B},
$$

where $P$ and $F$ are measures of Planarity and Filamentarity respectively $(P, F \leq 1)$. A sphere has $P=F=0$, an ideal filament has $P=0, F=1$ while $P=1, F=0$ for an ideal pancake. Other interesting shapes include 'ribbons' for which $P \sim F \sim 1$. When combined with the genus measure, the triplet $\{P, F, G\}$ provides an example of shape-space which incorporates information about topology as well as morphology of superclusters and voids.

Note however that non-geometrical shapefinding statistics based on mass moments etc. can give misleading results when applied to large scale structure, as demonstrated in [26].

\section{Numerical Estimates of Minkowski Functionals}

We construct a 2-polytopal surface using an assembly of triangles in which every triangle shares its sides with each of its three neighboring triangles. Here we outline the technique used for the numerical estimate of the Minkowski Functionals. It was suggested in [37], where it is described in more detail, see also [36]

- The area of such a triangulated surface is

$$
A=\sum_{i=1}^{N_{F}} A_{i}
$$

where $A_{i}$ is the area of the $i^{t h}$ triangle face and $N_{F}$ is the total number of faces which compose a given surface.
- The volume enclosed by this 2-polytopal surface is the summed contribution from $N_{F}$ tetrahedra

$$
\begin{aligned}
V & =\sum_{i=1}^{N_{F}} V_{i}, \\
V_{i} & =\frac{1}{3} A_{i}\left(n_{j} \bar{P}^{j}\right)_{i} .
\end{aligned}
$$

Here $V_{i}$ is the volume of an individual tetrahedron whose base is a triangle on the surface. $\left(n_{j} \bar{P}^{j}\right)$ is the scalar product between the outward pointing normal $\hat{n}$ to this triangle and the mean position vector of the three triangle vertices, for which the $j^{t h}$ component is given by

$$
\overline{\mathbf{P}}^{\mathbf{j}}=\frac{1}{3}\left(P_{1}^{j}+P_{2}^{j}+P_{3}^{j}\right) .
$$

The subscript $i$ in (10) refers to the $i$-th tetrahedron, while the vectors $P_{1}, P_{2}, P_{3}$ in (11) define the location of each of three triangle vertices defining the base of the tetrahedron relative to an origin. The origin can be arbitrary chosen.

- The extrinsic curvature of a triangulated surface is localized in the triangle edges. As a result the integrated mean curvature $C$ is determined by the formula

$$
C=\frac{1}{2} \sum_{i, j} \ell_{i j} \cdot \phi_{i j} \cdot \epsilon,
$$

where $\ell_{i j}$ is the length of the edge common to triangles $i$ and $j$ and $\phi_{i j}$ is the angle between the normals $\hat{n}_{i} \&$ $\hat{n}_{j}$ to these adjacent triangles

$$
\cos \phi_{i j}=\hat{n_{i}} \cdot \hat{n_{j}} \text {. }
$$

The summation in 12 is carried out over all edges. It should be noted that for a completely general surface, the extrinsic curvature can be positive at some (convex) points and negative at some other (concave) points on the surface.

- The Euler characteristics of a polyhedral surface is given by

$$
\chi=N_{F}-N_{E}+N_{V},
$$

where $N_{F}, N_{E}, N_{V}$ are, respectively, the total number of faces, edges, and vertices defining the surface. Formula 14 is correct for arbitrary shaped faces, obviously it can be reduced to

$$
\chi=N_{V}-N_{F} / 2,
$$

in the case when all faces are triangles since the number of edges is $N_{E}=3 N_{F} / 2$, which also holds true for polytopal surfaces.

\section{RESUlts}

In this section we present two set of tests focused on simple geometries and a more complex surface extracted from a cosmological N-body simulation. 

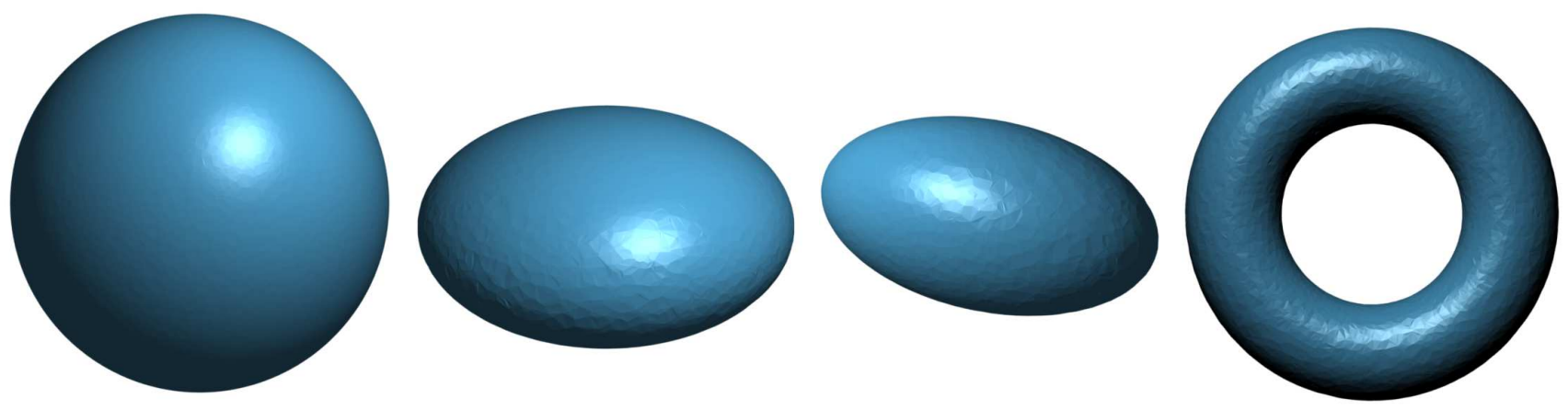

Figure 2. Four simple geometries used for testing, from left to right: sphere, pancake, filament and torus.

\section{A. Simple Models}

We constructed a set of simple geometries in order to test our method. Three geometries represent the generic types of the gravitational collapse in the Zel'dovich framework [31], [47]: a compact clump represented by a sphere, pancake and filament. Additionally we construct a torus in order to test its topology. The geometries were constructed by generating a set of hypothetical sampling points over a continuous scalar field defined as:

$$
d=\left(\frac{x^{2}}{a^{2}}+\frac{y^{2}}{b^{2}}+\frac{z^{2}}{c^{2}}\right)^{1 / 2} .
$$

Where $d$ is the scalar value of the field and we define the following limiting cases:

$$
\begin{array}{lll}
a=b=c & : & \text { sphere } \\
a \approx b \gg c & : & \text { pancake } \\
a \gg b \approx c & : & \text { filament. }
\end{array}
$$

The torus was computed as:

$$
d=\left(\left(R-\left(x^{2}+y^{2}\right)^{1 / 2}\right)^{2}+z^{2}\right)^{1 / 2} .
$$

Where $R$ is the radius of the torus and $t$ is the radius of the tube. We evaluated the distance function for each of the sampling points and proceed to construct the isosurface at a defined threshold value. Since the isosurfaces defined on the distance field enclose underdense regions we flip the normals so that they point outside the surface. Note that in this test surfaces the sampling points are not coupled with the scalar field. They are used only to define the tessellation that will be used to interpolate the density field.

Fig. 2 shows the four models after we extraced the isosurface. We rendered the raw surface (i.e. no normal smoothing) in order to emphasize the triangles that form the surface. The MTA produces very smooth surfaces although in this case it only reflects the spatial sampling of the random points.
Table I sumarizes the result of our analysis over the four geometries. The parameters for which there are analytical formulae (e.g. all parameters for the sphere) were computed with the accuracy better than $1 \%$. The numbers of faces, edges and vertices in the case of the sphere were 8,553, 25,653 and 17,102 . The numbers of the triangulation parameters in the cases of the filament, pancake and torus were similar while two largest superclusters in the cosmological $\mathrm{N}$-body simulation they were about and order of magnitude greater.

Table I

MINKOWSKI FUNCTIONALS AND SHAPE MEASURES

\begin{tabular}{lllll}
\hline \hline Mink Func & Sphere & Pancake & Filament & Torus \\
Volume & 0.065 & 0.016 & 0.004 & 0.059 \\
Area & 0.788 & 0.449 & 0.163 & 1.183 \\
C & 3.156 & 2.564 & 1.826 & 5.923 \\
EC & 2 & 2 & 2 & 0 \\
\hline Thickness & 0.248 & 0.109 & 0.075 & 0.149 \\
Breadth & 0.249 & 0.175 & 0.089 & 0.199 \\
Lenght & 0.251 & 0.204 & 0.145 & 0.471 \\
\hline Planarity & 0.002 & 0.233 & 0.086 & 0.143 \\
Filamentarity & 0.002 & 0.075 & 0.238 & 0.404 \\
\hline
\end{tabular}

\section{B. Cosmological Density Field}

We applied our method to a more realistic and complex dataset consistinng of a dark-matter N-body simulation of a standard $\Lambda \mathrm{CDM}$ cosmology. The simulation is contained inside a periodic box of $200 h^{-1} \mathrm{Mpc}$. Initial conditions were generated on a $256^{3}$ grid with $\Omega_{m}=0.3, \Omega_{\Lambda}=0.7$, $\sigma_{8}=0.9$ and $H=73 \mathrm{~km} \mathrm{~s}^{-1} \mathrm{Mpc}^{-1}$. 1 After having set up the initial conditions, we follow the subsequent gravitational evolution to the present time using the public N-body code Gadget 2 [40]. The simulation box is large enough to contain the full range of environments in the Cosmic Web, from large underdense voids to massive dense clusters.

\footnotetext{
${ }^{1}$ One megaparsec (Mpc) corresponds to $3.26 \times 10^{6}$ light years, $\Omega_{m}$ and $\Omega_{\Lambda}$ correspond to the ratio of matter and dark energy relatively to a close universe with $\Omega_{\text {total }}=1$. The Hubble constant $H$ measures the expansion of the Universe as a recession velocity per Mpc.
} 

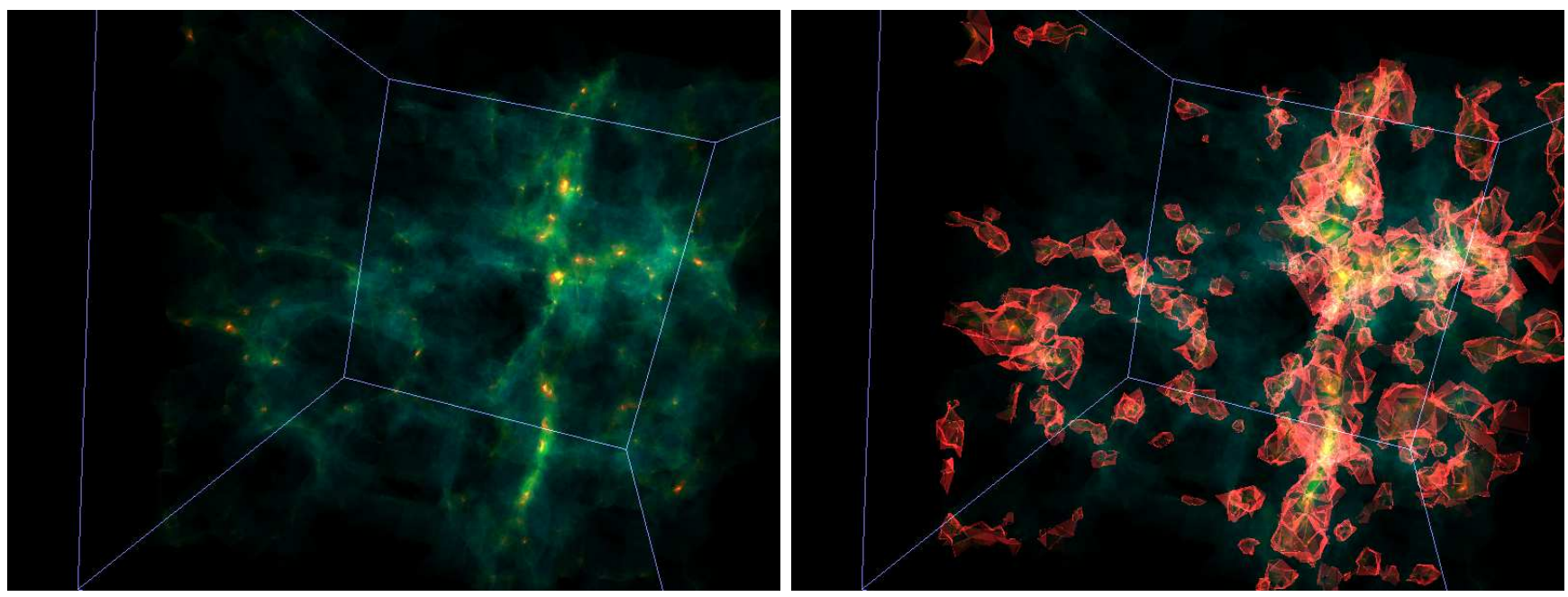

Figure 3. Volume rendering of a density field (left) and isodensity surface at $\delta=3$ (right).

Table II

MINKOWSKI FUNCTIONALS AND SHAPE MEASURES OF TWO LARGEST SUPERCLUSTERS IN THE N-BODY SIMULATION

\begin{tabular}{lll}
\hline \hline Mink Func & Green & Red \\
\hline Volume & 0.00359 & 0.00202 \\
Area & 0.429 & 0.234 \\
C & 13.34 & 6.54 \\
EC & -10 & -6 \\
\hline Thickness & 0.0251 & 0.0258 \\
Breadth & 0.0322 & 0.0358 \\
Lenght & 1.06 & 0.521 \\
\hline Planarity & 0.124 & 0.162 \\
Filamentarity & 0.941 & 0.871 \\
\hline
\end{tabular}

From the final particle distribution we estimate the local density using a Gaussian kernel of $2 \mathrm{Mpc} / \mathrm{h}$. We then compute the Delaunay tessellation with periodic conditions and applied the MTA (section $\amalg$ in order to construct an isodensity surface corresponding to an overdensity threshold of $\delta=3$. From the raw isosurface we label individual objects as described in section $\amalg I I-\mathrm{A}$ and compute their MF and shapefinders (see section IV-A). Fig. 3 shows the volume rendering of the density field inside the simulation box (left panel) and the isosurface extracted at $\delta=3$ (right panel). The isosurface is formed by a large number of unconnected structures enclosing overdense regions and clumps of matter.

Fig. 4 shows the two largest superclusters depicted from the N-body simulation. The largest structure is shown in blue and the second largest in red. Their parameters, MF, thickness, breadth, length and shapefinders are given in Table [II Both have comparable thicknesses and breadths while the length of the first largest supercluster about twice longer than that of the second largest cluster. The superclusters have 6 and 4 tunnels respectively, a couple of which can be seen in the figure. Although the figure gives some idea about the complexity of the objects, its full appreciation is possible only with the help of visualization software where the threedimensional structure of each can be fully recognized.

\section{CONCLUSION AND FUTURE WORK}

We present a novel technique for computing Minkowski functional i.e. the volume, area, integrated mean curvature and Euler characteristic of complex objects (superclusters and voids of galaxies) that form due to the nonlinear gravitational growth of initial Gaussian random fluctuations in dark matter density field. These structures can be reliably modeled in cosmological N-body simulations and are observed in large galaxy redshift catalogs like SDSS and $2 \mathrm{dF}$. The technique can also be used for other applications when quantitative information is needed for arbitrary shaped structures. An important step forward has been made by replacing the Marching Cube Algorithm in previous studies [34], [35], [37] by Marching Tetrahedra Algorithm for building the isodensity surface. The MCA operates on the fields specified on a uniform cubic grid and therefore has a uniform spatial resolution. The MTA finds the density field directly from the positions of galaxies or particles in cosmological N-body simulations by means of Delaunay tessellation and therefore has an adaptive spatial resolution. Potentially the galaxy density field can be analyzed with better resolution in the regions where more galaxies are observed or more simulation particles are clustered however this requires additional testing which under way and will be reported in the following papers.

In this work we report the results of the basic tests that shows that the code works and ready for the studies of the density fields obtained in cosmological N-body simulations of the structure in the universe or real structures in galaxy redshift catalogs. 

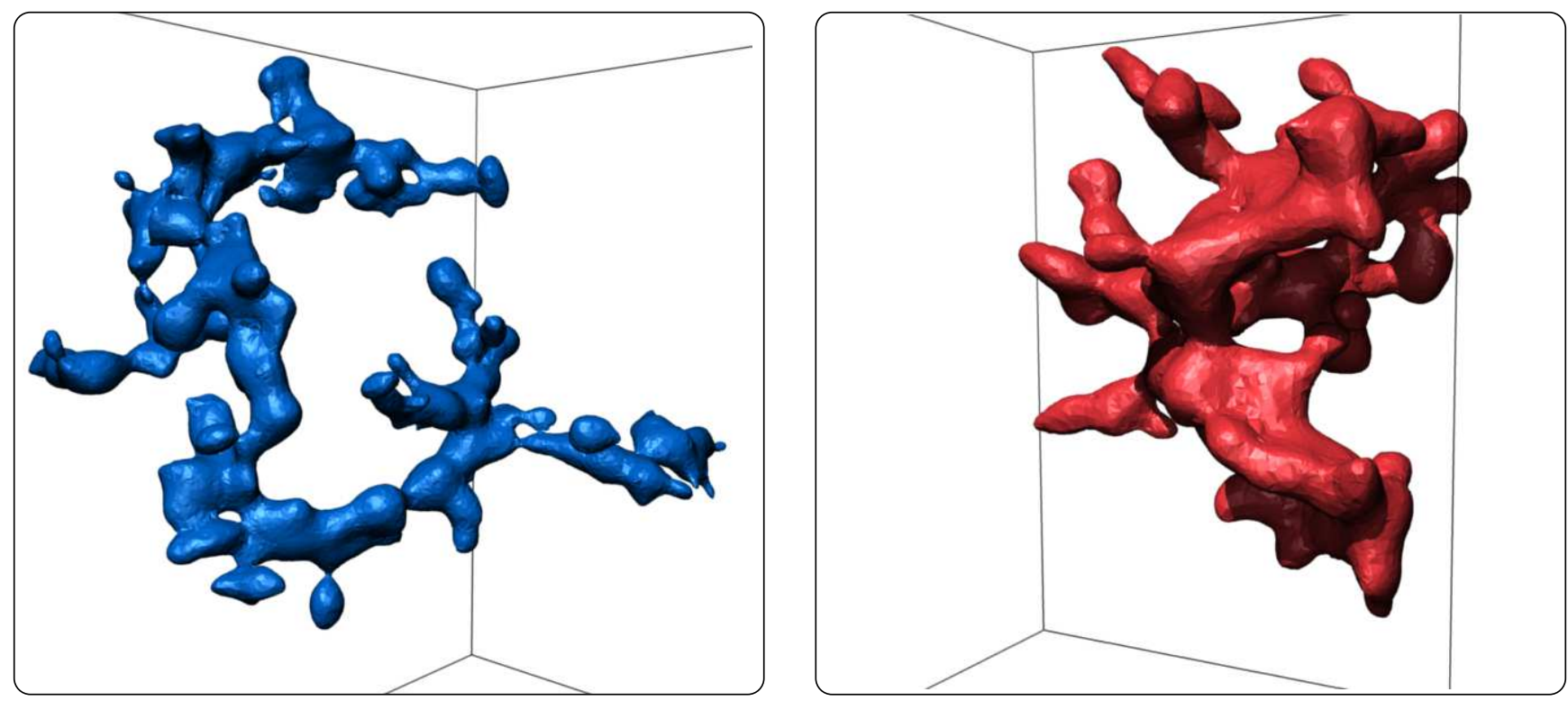

Figure 4. Largest (blue) and second largest (red) structures at $\delta_{t}=3$ and Gaussian smoothing of $2 \mathrm{Mpc} / h$.

\section{REFERENCES}

[1] Aragón-Calvo M. A., Jones B. J. T., van de Weygaert R., van der Hulst J. M. 2007, AAP, 474, 315

[2] Aragón-Calvo M. A., Platen, E.., van de Weygaert R., Szalay, A., arXiv:0809.5104 Unpublished.

[3] Bardeen J. M., Bond J. R., Kaiser N., Szalay A. S. 1986, The Astrophysical Journal, 304, 15

[4] Barrow, J.D., Sonoda, D.H. \& Bhavsar, S.P., 1985, Monthly Notices of the Royal Astronomical Society, 216, 17

[5] Bernardeau, F., \& van de Weygaert, R. 1996, Monthly Notices of the Royal Astronomical Society , 279, 693

[6] Bertschinger E., 1987, The Astrophysical Journal, 323, 103

[7] P.-T. Bremer, H. Edelsbrunner, B. Hamann, and V. Pascucci. A topological hierarchy for functions on triangulated surfaces. IEEE Transactions on Visualization and Computer Graphics, 10(4):385396, 2004

[8] Coles, P., \& Jones, B. 1991, Monthly Notices of the Royal Astronomical Society , 248, 1

[9] Delaunay B., 1934, Bull. Acad. Sci. USSR (VII) Classe Sci. Mat., pp 793-800.

[10] A. Doi, A. Koide, An Efficient Method of Triangulating Equivalued Surfaces by using Tetrahedral Cells. IEICE Transactions Communication, Elec. Info. Syst, E74(1) 214-224, January 1991

[11] H. Edelsbrunner, J. Harer, V. Natarajan and V. Pascucci. Morse-Smale complexes for piecewise linear 3-manifolds. In Proc. 19th Ann. Sympos. Comput. Geom., pages 361370, 2003
[12] Gott III, J.R., Melott, A.L., Dickinson, M. 1986, The Astrophysical Journal, 306, 341

[13] A. Gueziec, R. Hummel, Exploiting Triangulated Surface Extraction using Tetrahedral Decomposition. IEEE Transactions on Visualisation and Computer Graphics, 1 (4) 328-342, December 1995

[14] Hahn O., Porciani C., Carollo C. M., Dekel A. 2007, Monthly Notices of the Royal Astronomical Society , 375, 489

[15] Janes, L.G. and Demarque, P., 1983, The Astrophysical Journal, 264, 206

[16] W. Lorensen, H. Cline, Marching Cubes: A High Resolution 3D Surface Construction Algorithm. Computer Graphics, 21 (4): 163-169, July 1987

[17] de Lapparent, V., Geller, M.J. \& Huchra, J.P., 1991, ApJ, 369, 273

[18] Malik, R. K. and Subramanian, K., 1997, Astronomy and Astrophysics, 317, 318.

[19] Matsubara, T., 2003, ApJ, 584, 1

[20] Mecke, K.R., Buchert, T. \& Wagner, H., 1994, Astronomy and Astrophysics 288, 697

[21] Novikov, D., Colombi, S. and Doré, O., 2006, Monthly Notices of the Royal Astronomical Society, 366, 1201

[22] Novikov, D.I., Feldman, H. and Shandarin, S.F., 1999 Int. J. Mod. Phys.D, 8, 291

[23] Okabe A., Boots B., Sugihara K., Chiu S. N., 2000, Spatial Tessellations, Concepts and Applications of Voronoi Diagrams. John Wiley, Chichester. 
[24] Peebles, P.J.E. 1980, The Large-Scale Structure of the Universe(Princeton : Princeton Univ. Press)

[25] Sahni, V., Sathyaprakash, B.S. \& Shandarin, S.F., 1998, The Astrophysical Journal Letters, 495, L5

[26] Sathyaprakash, B.S., Sahni, V. \& Shandarin, S.F., 1998, The Astrophysical Journal, 508, 551

[27] Schaap W. E., van de Weygaert R. 2000, Astronomy and Astrophysics, 363, L29

[28] Schaap, W. E. 2007, The Delaunay Tessellation Field Estimator, Ph.D. Thesis, University of Groningen

[29] Schmalzing, J. \& Buchert, T., 1997, The Astrophysical Journal Letters, 482, L1

[30] Shandarin, S.F. 1983 Sov. Astron. Lett., 9, 104

[31] Shandarin, S.F. and Zeldovich, Ya.B. 1989, Rev.Mod.Phys.,61, 185.

[32] Shandarin, S., Habib, S., Heitmann, K. 2009, arXiv:0912.4471

[33] Shandarin, S.F., Feldman, H.A., Xu, Y., \& Tegmark, M. 2002, ApJS, 141, 1

[34] Shandarin, S.F., Sheth, J., Sahni, V. 2004, Monthly Notices of the Royal Astronomical Society, 353, 162

[35] Sheth, J.V. 2004a, Monthly Notices of the Royal Astronomical Society, 354, 332

[36] Sheth, J.V. 2004b, PhD Thesis, Pune University, Pune, India

[37] Sheth, J.V., Sahni, V., Shandarin, S.F. \& Sathyaprakash, B. 2003, Monthly Notices of the Royal Astronomical Society, 343,22

[38] Sibson R., 1981, In: Barnet V.(ed.) Interpreting Multivariate Data. Wiley, Chichester

[39] Sousbie, T., Pichon, C., Colombi, S., Novikov, D., and Pogosyan, D. 2008, Monthly Notices of the Royal Astronomical Society, 383, 1655

[40] Springel V., White S.D.M., Jenkins A., Frenk C.S., Yoshida N., Gao L., Navarro J., Thacker R., Croton D., Helly J., Peacock J.A., Cole S., Thomas P., Couchman H., Evrard A., Colberg J.M., Pearce F. 2005, Nature, 435, 629

[41] Sugiyama, N. 1995, ApJS, 100, 281

[42] Sukumar N., 1998, PhD thesis, Northwestern University.

[43] van de Weygaert, R., Icke, V. 1989, AAP, 213, 1.

[44] van de Weygaert R., Schaap W.E 2009, The Cosmic Web: Geometric Analysis. In: Data Analysis in Cosmology, eds. V. Martínez et al., Lecture Notes Phys., 665, 291-413 (SpringerVerlag) arXiv:0708:1441

[45] Voronoi G., 1908, J. Reine Angew. Math., 134, 198
[46] Watson D. F., 1992, Contouring: A Guide to the Analysis and Display of Spatial Data. Pergamon Press.

[47] Zel'dovich, Ya.B. 1970, Astronomy and Astrophysics, 5, 84

[48] Zel'dovich, Ya.B., Einasto, J. and Shandarin, S.F., 1982, Nature, 300, 407 